\title{
A Cross Entropy-Based Heuristic for the Capacitated Multi-Source Weber Problem with Facility Fixed Cost
}

\author{
Seyed Javad Hosseininezhad ${ }^{1}$, Said Salhi ${ }^{2}$, Mohammad Saeed Jabalameli ${ }^{3}$
}

\author{
${ }^{1}$ Department of Industrial engineering, K. N. Toosi University of Technology, Tehran, Iran \\ ${ }^{2}$ The Centre for Logistics and Heuristic Optimisation, Kent Business School, University of Kent at Canterbury, Canterbury CT2 \\ 7PE, UK \\ ${ }^{3}$ Department of Industrial engineering, Iran University of Science and Technology, Tehran, Iran
}

\begin{abstract}
This paper investigates a capacitated planar location-allocation problem with facility fixed cost. A zonebased fixed cost which consists of production and installation costs is considered. A nonlinear and mixed integer formulation is first presented. A powerful three stage Cross Entropy meta-heuristic with novel density functions is proposed. In the first stage a covering location problem providing a multivariate normal density function for the associated stochastic problem is solved. The allocation values considering a multinomial density function are obtained in the second stage. In the third stage, single facility continuous location problems are solved. Several instances of various sizes are used to assess the performance of the proposed meta-heuristic. Our approach performs well when compared with the optimizer GAMS which is used to provide the optimal solution for small size instances and lower/upper bounds for some of the larger ones.
\end{abstract}

Keywords: Meta-heuristics, Evolutionary methods, Planar location, Cross Entropy, GAMS.

\section{Introduction}

In this study, we are interested in determining the optimal or best location of $m$ capacitated facilities in the continuous space with the presence of zone-based facility fixed cost. The objective is to minimize the total cost which includes the cost of transportation, installation, production and the cost for the unmet demand. This new logistical problem is first formulated as a nonlinear and mixed integer programming problem. The software GAMS is used to find optimal solution for small/medium size instance and also to provide lower/upper bounds for the larger ones. A new Cross Entropy meta-heuristic based on three stages is then proposed using appropriate density functions. 
We briefly review those recent papers that address planar location problems that are closely related to ours. Since the planar location-allocation (LA) problem, also known as the Multi-Source Weber problem (MSWP), was proposed in the sixties by Cooper (1963), recent advances are put forward including among others Shöbel and Scholz (2010), and Brimberg and Drezner (2013). The former proposed a new approach which they call the Big Cube Small Cube to optimally solve the 2 and 3 facilities in higher dimensions. This is based on the Big Square Small Square method originally given by Hansen et al. (1981). The latter developed an improved implementation of Cooper's alternate locate allocate algorithm which is further enhanced by a transfer follow-up. When the facilities have a limited capacity, the problem becomes the capacitated MSWP. Brimberg et al. (2014) proposed a new local search approach is embedded within Variable Neighborhood Search for solving the multi-source Weber problem. The algorithm switches between the continuous model and its discrete counterpart until no further improvement can be found in either. Luis et al. (2009) provided constructive and adaptive heuristics to generate initial solutions. . Mohammadi et al. (2010) used a new method that uses two genetic algorithms for capacitated multi source Weber problem. The first, solves the location problem while the second, solves the allocation problem. A GRASP-based heuristic was also proposed by Luis et al. (2011) where adaptive learning is used to construct the restricted candidate list. Akyüzet al. (2013) proposed two types of branch and bound algorithms for the capacitated MSWP. The first is an allocation space based-branch and bound algorithm whereas the second is based on the partition of the location space.

Several researches are also in uncertain environment. Durmazet al. (2009) used a discrete approximation technique to address this problem with probabilistic customer locations Mousavi and AkhavanNiaki (2013) studied a capacitated location allocation problem with stochastic location and fuzzy demand. Three fuzzy programming models were developed and a hybrid intelligent algorithm was provided to solve the problem.

The above location allocation problems do not consider fixed cost in the continuous space. However, there are situations in practice which may include zones with high installation cost. These can be modeled using fixed cost. Brimberg et al. (2004) introduced the multi-source Weber problem with constant opening cost in the continuous space. They developed a solution method uses a multi-phase heuristic that first solves a discrete version of the problem by existing methods to obtain an estimate of the optimal number of facilities. Brimberg and Salhi (2005) are among the ones that introduced a zone-dependent fixed cost for the incapacitated single facility location-allocation problem in the continuous space. An efficient algorithm that determines the solution optimally was proposed. The authors also provide a simple but an informative illustrative example. For a review on the continuous location problem in general, see Drezner et al. (2001), and Brimberget al. (2008). 
To the best of our knowledge, this is the first time the meta-heuristic Cross Entropy (CE) is developed to solve this complex continuous location problem.

The remainder of the paper is organized as follows. In the next section, a nonlinear and integer programming formulation is proposed. A meta-heuristic using a Cross Entropy algorithm is presented in section 3. Computational results are given in section 4. Sensitivity Analysis is provided in section 5. The last section summarizes our conclusions and highlights some research areas.

\section{A Capacitated Multi-Source Weber Problem with Facility Fixed Cost}

Consider there are $n$ customers (demand points) indexed by $i$ and $m$ facilities indexed by $j$. The following notation is used. We first divide the space into $n$ zones and introduce a binary variable $z_{j i}$ that defines whether or not facility $j$ is located in zone $i$. It is assumed that the distance between each customer and a facility is Euclidean. We formulate the problem as a $0-1$ nonlinear and mixed integer programming model. We first provide the necessary notations followed by the mathematical formulation.

\section{Notation}

\section{Sets/Indices}

$N$ set of zones (demand points) in the continuous space indexed by $i,\{i=1,2, \ldots, n\}$

$K$ set of new facilities to be located indexed by $j,\{j=1,2, \ldots, m\}$

\section{Parameters}

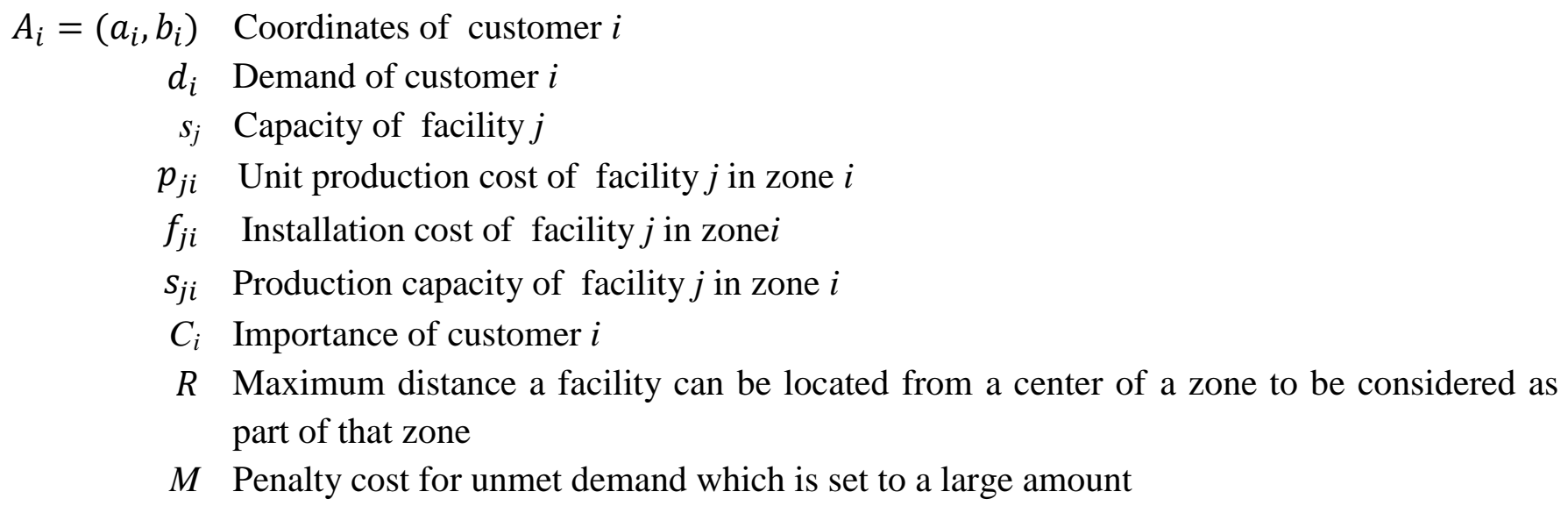

\section{Decision variables}

$$
\begin{array}{rl}
X_{j}=\left(x_{j}, y_{j}\right) & \text { Coordinate of facility } j \text { in the plane } \\
D\left(X_{j}, A_{i}\right) & \text { distance between customer } i \text { and facility } j \\
T_{j i} & \text { amount supplied from facility } j \text { to customer } i \\
z_{j i} & 1 \text { if facility } j \text { is located in zone } i \text {; } 0 \text { otherwise } \\
q_{i} & \text { Unmet demand of customer } i\left(q_{i} \leq d_{i}\right)
\end{array}
$$




\section{Formulation}

$\min \sum_{i=1}^{n} \sum_{j=1}^{m} T_{j i} \cdot D\left(X_{j}, A_{i}\right)+\sum_{j=1}^{m} \sum_{i=1}^{n} z_{j i} p_{j i} \sum_{i=1}^{n} T_{j i}+\sum_{j=1}^{m} \sum_{i=1}^{n} z_{j i} f_{j i}+M \sum_{i=1}^{n} C_{i}\left(\frac{q_{i}}{d_{i}}\right)$

Subject to

$\sum_{i=1}^{n} z_{j i} \cdot D\left(X_{j}, A_{i}\right) \leq R, \quad \forall j=1,2, \ldots, m$

$\sum_{i=1}^{n} z_{j i}=1, \quad \forall j=1,2, \ldots, m$

$\sum_{j=1}^{m} z_{j i} \leq 1, \quad \forall i=1,2, \ldots, n$

$\sum_{i=1}^{n} T_{j i} \leq \sum_{i=1}^{n} z_{j i} s_{j i}, \quad \forall j=1,2, \ldots, m$

$\sum_{j=1}^{m} T_{j i}+q_{i}=d_{i}, \quad i=1,2, \ldots, n$

$T_{j i} \geq 0, z_{j i} \in\{0,1\}, q_{i} \geq 0, X_{j}=\left(x_{j}, y_{j}\right) \in \mathbb{R}^{+} \quad \forall i, j$

Equation (1) is the objective function which is made up of four terms. These include the transportation cost, the production cost, the installation cost and the cost associated with the unmet demand. Constraint set (2) guarantees that if the distance between facility $j$ and zone $i$ is greater than $\mathrm{R}$, then $z_{j i}=0$ (i.e., facility $j$ does not belong to zone $i$ ), otherwise $z_{j i}=1$. Constraint set(3) states that facility $j$ is installed only in one zone whereas constraint set (4) shows that only one facility could be located in any zone at most. Constraint set (5) is the capacity constraint (i.e., the production at facility $j$ is not violated in zone $i$ ) and constraint set (6) guarantees that the sum of the assigned and the unmet demand at each customer is equal to the demand of that customer.

This formulation is used in the optimizer GAMS to produce the optimal solution for small size problems, and lower and upper bounds for the larger ones. These results will be used to evaluate the performance of a solution method based on the evolutionary meta-heuristic Cross Entropy (CE) which we propose next.

\section{A Cross Entropy solution method}

In this section we first provide the basic CE algorithm which serves as a basis for our new CE metaheuristic. This is followed by a subsection explaining some of the main steps of the algorithm. 


\subsection{The basic CE algorithm}

The main idea of Cross Entropy (CE) was originally introduced by Rubinstein (1997). CE is an evolutionary technique that is related to the design of an effective learning mechanism which is used throughout the search. For background knowledge, applications and recent references on Evolutionary methods, the reader may consult the interesting paper on location-routing by Prodhon (2011). The main principle of $\mathrm{CE}$ is to associate an estimation problem to the original combinatorial optimization problem. This is called the associated stochastic problem which is characterized by a density function $\phi$. This stochastic problem is then solved by identifying the optimal sampling density $\phi^{*}$, which is the one that minimizes the Kullback-Leibler distance with respect to the original density $\phi$. This distance is also known as the CE between $\phi$ and $\phi^{*}$. The minimization of the CE leads to the construction of "optimal" updating rules for the density function, and consequently to the generation of improved feasible solutions. The method terminates when the convergence to a point in the feasible region is achieved. The main features of the $C E$ algorithm were discussed by de Boer et al. (2005). The application of $C E$ in the area of optimization can be found in Kroese and Rubinstein (2005). Specific combinatorial problems such as the max-cut problem, the travelling salesman problem and the capacitated vehicle routing problem were solved by Rubinstein and Kroese (2004). A new meta-heuristic scheme for the Integer Knapsack Problem with setups was presented by Caserta et al. (2008). The authors proposed a CE based scheme with an "intelligent" mechanism aimed at choosing the items to be in the knapsack. Caserta and Quinonez Rico (2009) developed a Lagrangean-based method that transforms the capacitated problem into a set of disjoint uncapacitated lot-sizing problems that are solved by an efficient $C E$-based algorithm. Recently, Bekker and Aldrich (2011) applied CE for multi-objective optimization problems. A classical CE algorithm is given in Algorithm 1.

Algorithm 1: A Cross Entropy (CE) for optimization problems

Step 1: Define the initial parameters of the density function $\phi$ for the associated stochastic problem.

Step 2: $\quad$ Generate a solution vector $\left(X_{1}, \ldots, X_{N}\right)$ for the problem via Monte Carlo simulation based on the density function $\phi$.

Step 3: Calculate the objective function of the problem for each of the solution in $\left(X_{1}, \ldots, X_{N}\right)$ and select the best solution.

Step 4: $\quad$ Update the parameters of the density function $\phi$ based on the best solution.

Step 5: If the stopping criterion is satisfied stop, otherwise go to Step 2. 


\section{2.The proposed CE algorithm}

In this paper, we present a $C E$ algorithm to solve the capacitated multi-source Weber problem defined in Section 2 (Eqs. 1-6). We consider two density functions to generate vectors for identifying the location and the customer allocation for each facility (Step 2). Let us define a family of density functions $\phi$ on $X$, and use a 2-dimensional multivariate normal density function for locating facilities under the following probability distribution function:

$$
\phi(X, \mu, \Sigma)=e^{-(X-\mu) \Sigma^{-1}(X-\mu)^{T} / 2} / \frac{1}{\Sigma^{1 / 2} 2 \pi}
$$

Where $X=(x, y)$ is 1-by-2 vector representing the $x$ and $y$ coordinates of facility locations, $\mu=\left(\mu_{x}, \mu_{y}\right)$ is the mean of facility locations and $\Sigma$ is a 2-by-2 covariance matrix which is a symmetric positive definite matrix.

We estimate $X$ via Monte Carlo simulation. In this regard, $X$ could be estimated by drawing a random sample $X^{1}, \ldots, X^{N}$ from $\phi(X, \mu, \Sigma)$. Hence, the multinomial density function is applied for allocating customers to facilities under the following probability distribution function:

$$
\phi(T, s, p)=\frac{s !}{T_{1} ! \cdots T_{n} !} p_{1} \cdots p_{n}
$$

Where $s$ is an integer number, $T=\left(T_{1}, \ldots, T_{n}\right)$ denotes the $1-b y-n$ vectors of the allocation values and $p=\left(p_{1} \ldots p_{n}\right)$ is the probabilities of allocating facilities to $n$ customers. We wish to estimate $T$ via Monte Carlo simulation by drawing a random sample $T^{1}, \ldots, T^{N}$ from $\phi(T, s, p)$, where $N$ is the $C E$ population size. At each iteration, the best solutions (elites) found in the previous iterations are also used to update the parameters of the associated probability distribution function. This updating scheme is given next.

The proposed $C E$ is made up of three stages. In the first stage, we consider the problem as a continuous covering location problem and find the location of the facilities using (7) until one of the stopping criteria is met. This includes the convergence or the maximum number of iterations whichever comes first. In the second stage, we solve the allocation problem based on the location found in the first stage using (8). Finally, in stage three we solve $m$ separate single continuous facility location problems while considering the allocation already found.

\subsection{Explanation of the main steps}

In Stage I, a continuous covering location problem is solved to determine the location of the facilities.

In the proposed problem, customer $i$ is assigned to facility $j$ if $D\left(X_{j}, A_{i}\right) \leq C R$ with $C R$ being a predetermined covering radius. At each iteration the covering matrix is computed. The error value is 
obtained based on the variance between the best obtained locations for each facility at iteration $t$. The mean of the errors for all facilities is calculated as the error value at iteration $t$. Also, in Stage II, the error value is obtained based on the difference between the probability of allocating the demands to facility $j$ at iterations $t$ and $t-1$.

The mean of the errors for all facilities is then calculated as the error value at iteration $t$. In Stage III, a post optimization is carried out based on Cooper's Alternating Transportation-Location Heuristic (Cooper, 1972). Since the allocation values (i.e., the value of $q_{i}$ ) and the cost representing the unmet demand are determined in phase II,our problem reduces to solving $m$ independent continuous location problems with the aim of minimizing the total cost. We define for each facility $j$, the cost "FacilityCost ${ }_{j}$ " as follows:

$$
\text { FacilityCost }_{j}=\sum_{i=1}^{n} T_{j i} \cdot D\left(X_{j}, A_{i}\right)+\sum_{i=1}^{n} z_{j i} p_{j i} \sum_{i=1}^{n} T_{j i}+\sum_{i=1}^{n} z_{j i} f_{j i} \forall j=1, \ldots, m
$$

Let FacilityCost ${ }_{j}^{1}$ and FacilityCost ${ }_{j}^{2}$ be the cost of facility $j$ before and after the implementation of phase III, respectively. The well known Weiszfeld equations are then used to update the new location of facility $j$ :

$$
x_{j}^{(g)}=\frac{\sum_{i=1}^{n} w_{j i} a_{i} / D\left(X_{j}^{(g-1)}, A_{i}\right)}{\sum_{i=1}^{n} w_{j i} / D\left(X_{j}^{(g-1)}, A_{i}\right)}, \quad \text { and } \quad y_{j}^{(g)}=\frac{\sum_{i=1}^{n} w_{j i} b_{i} / D\left(X_{j}^{(g-1)}, A_{i}\right)}{\sum_{i=1}^{n} w_{j i} / D\left(X_{j}^{(g-1)}, A_{i}\right)},
$$

Where $X_{j}^{(g)}=\left(x_{j}^{(g)}, y_{j}^{(g)}\right)$ denotes the location of facility $j$ at iteration $g$ and $A_{i}=\left(a_{i}, b_{i}\right)$ the coordinates of customer $i$. This procedure is carried out until there is no significant change in the location of facility $j$. The corresponding values of $z_{j i}$ are then determined and the fixed costs including the production cost and the installation cost for facility $j$ are computed leading to the evaluation of FacilityCost $_{j}$ in (9).

In summary, for each facility $j=1, \ldots, m$, if FacilityCost $_{j}^{2}<$ FacilityCost $_{j}^{1}$, the new location $j$ is selected, otherwise the previous location is retained. A pseudo-code of the proposed Cross Entropy (CE) algorithm is given in Algorithm 2. 
Algorithm 2: Cross Entropy (CE) for the Capacitated Multi-Source Weber Problem

1: Determine the population size and the number of elite solutions, error ${ }^{0}$ (initial error), $\varepsilon($ Min error) and maxiter (the max number of iterations),

\section{Stage I: (Solving the continuous covering problem)}

\section{Repeat}

2: Generate the initial multivariate normal distribution parameters (Mean and covariance) based on the $x$ and $y$ coordinates of the zones.

Draw a sample population $X^{1}, \ldots, X^{N} \sim \operatorname{MV} \operatorname{Normal}\left(X, \mu_{x}^{t}, \mu_{y}^{t}, \Sigma^{t}\right)$.

3: For each random vector $X^{l}=\left(x_{l}, y_{l}\right)(l=1, \ldots, N)$, solve the model as a continuous covering location problem

4: $\quad$ Select the best solution of the sample population(elites) as $X_{\text {elite }}^{t}=\left(x_{\text {elite }}^{t}, y_{\text {elite }}^{t}\right)$

5: $\quad$ Update $\mu_{x}, \mu_{y}$ and $\Sigma$ as follows:

$$
\begin{aligned}
& \mu_{x}^{t}=\operatorname{mean}\left(x_{\text {elite }}^{t}\right) \\
& \mu_{y}^{t}=\operatorname{mean}\left(y_{\text {elite }}^{t}\right) \\
& \Sigma^{t}=\operatorname{covariance}\left(x_{\text {elite }}^{t}, y_{\text {elite }}^{t}\right) \\
& e_{j}^{t}=\operatorname{variance}\left(x_{\text {elite }}^{t}, y_{\text {elite }}^{t}\right)_{j}, \quad \forall j=1, \ldots, m \\
& \text { error }{ }^{t}=\operatorname{mean}\left(e_{j}^{t}\right)
\end{aligned}
$$

Until ( error $^{t} \leq \varepsilon \vee t>$ maxiter) $t \leftarrow t+1$

\section{Stage II: (Solving the allocation problem)}

\section{Repeat}

6: $\quad$ Generate initial multinomial distribution probabilities for allocating demands $\left(P^{0}\right)$ and draw a sample population $T^{1}, \ldots, T^{N} \sim \operatorname{MNomial}\left(P^{t}\right)$.

7: For each random vector $T^{l}(l=1, \ldots, N)$ solve the Capacitated Multi-Source Weber Problem.

8: $\quad$ Select the best solution for the sample population(elites)

9: $\quad$ Update $P^{t}$ as follows $\left(T_{j i}^{t, e l i t e}\right.$ denotes the best allocation values from facility $j$ to customer $i$ at iteration $t)$ :

$$
\begin{aligned}
& p_{j}^{t}=\left(p_{j 1}^{t}, \ldots, p_{j n}^{t}\right)=\left(T_{j 1}^{t, \text { elite }} / \sum_{i} T_{j 1}^{t, \text { elite }}, \ldots, T_{j n}^{t, \text { elite }} / \sum_{i} T_{j i}^{t, \text { elite }}\right), \forall j=1, \ldots, m \\
& e_{j}^{t}=p_{j}^{t}-p_{j}^{t-1} \quad \forall j=1, \ldots, m \\
& \operatorname{error}^{t}=\operatorname{mean}\left(e_{j}^{t}\right)
\end{aligned}
$$

Until ( error $^{t} \leq \varepsilon \vee t>$ maxiter) $t \leftarrow t+1$

\section{Stage III: (Solving the facility location problem)}

10: For each facility $j$ ( $m$ separate single facility location problem)

Calculate "FacilityCost ${ }_{j}^{1}\left(X_{j}^{(\text {Stage I })}, T_{j}^{(\text {Stage II })}\right)$ ", where $X_{j}^{(\text {Stage I) }}$ is the location value obtained in Stage I and $T_{j}^{(\text {Stage I) }}$ is the allocation values obtained in Stage II for facility $j$.

\section{Repeat}


Update $X_{j}^{(g)}$ based on the Weiszfeld equations ( $\delta$ is Min error):

Until $\left(X_{j}^{(g)}-X_{j}^{(g-1)} \leq \delta\right) g \leftarrow g+1$

Calculate "FacilityCost ${ }_{j}^{2}\left(X_{j}^{(g)}, T_{j}^{(\text {Stage II })}\right)$ " value for facility $j$.

if FacilityCost $t_{j}^{2}<$ FacilityCost ${ }_{j}^{1}$ then $X_{j}^{*}=X_{j}^{(g)}$ else $X_{j}^{*}=X_{j}^{(\text {Stage I) }}$ (where $X_{j}^{*}$ is the best location value for facility $j$ )

\section{Computational results}

The $C E$ algorithm is implemented in Matlab and run on a Core i5 at $2.53 \mathrm{GHz}$ with $3 G B$ of $R A M$ memory. The CE parameters for Stage I and Stage II are the following: (population size, elite size $)=(300$, $15)$ and $(500,25)$, respectively. The covering radius for stage $I$ is $1.5, R=0.4$ and $M=10,000$. We tested our approach in small/medium size instances ( $n=20$ to 100 with a step size of 20) and large ones $(n=200,300$, $400,500,1000)$. The customers are located in the grids of the rectangular areas that depend on the value of $n$. For example for $n=20$, the $(x-y)$ coordinates of the 20 customers are generated at the grid points of the $5 \times 4$ rectangle where customer 1 is at position $(1,1)$, customer 2 at $(2,1)$, etc. The remaining instances are generated in the same way using the following rectangles: $6 \times 5(n=30), 8 \times 5(n=40), 10 \times 10(n=100)$, $20 \times 10(n=200), 20 \times 15(n=300), 20 \times 20(n=400), 25 \times 20(n=500), 40 \times 25(n=1000)$. The demand of the customers is randomly generated in the range $[1,10]$. The capacity of the facilities is based on the size of the problem and it is randomly generated in the range [45, 60]. As we introduced the cost for the unmet demand, we constrained the choice of the capacities in such a way that the sum of the capacities of all the open facilities is smaller than the total customers demand. The fixed cost is randomly generated in the range [800,1000]. The importance of each customer and the unit production cost are both set to 1 .

We carried out our experiments using two scenarios. The first one deals with small/medium size instances whereas the second treats the large instances. We compare the obtained $C P U$ time and the objective function value of the proposed algorithm with GAMS software using Baron Solver. We ran the algorithms ten times for each instance and report the following statistics. These include the average (Aver), best value (Best), standard deviation (Std) and the Coefficient of Variation (CV) of the objective function found. The percent deviation from the best-known solutions found by GAMS is also reported as (Dev) which is computed as follows:

$$
D e v=\frac{F^{\text {best }}-F^{*}}{F^{*}} \times 100
$$

Where $F^{\text {best }}$ is the total cost found by the proposed (CE) algorithm and $F^{*}$ refers to the cost of the best solution (or the optimal/upper bound) found by GAMS within the allowed $C P U$ time. 
Scenario 1 (small/medium size instances)

In this experiment, we report the results for $n=20$ to 100 , with $m=2$ to 10 . GAMS is used to yield optimal solutions. According to Table 1, the proposed CE algorithm provides solutions that are close to those obtained by the optimizer GAMS, with deviations being less than $2 \%$ in most cases. In addition, our heuristic requires a tiny fraction of the $C P U$ time used by GAMS.

\section{Scenario 2 (Large size instances)}

We applied our CE algorithm for large instances up to $n=1000$ and $m=100$, see Table 2. Though GAMS showed to be relatively slower, we let it run for up to 5 hours so we can report Lower Bound (LB) and Upper Bound (UB).The deviation from the UB value and the $C P U$ time if the solution is found within the set time of $5 \mathrm{hrs}$ are also reported in Table 2. In summary, the proposed $C E$ algorithm outperformed the optimizer GAMS which, in most cases, failed to provide values for $L B$ or $U B$ within the allowed $C P U$ time.

TABLE 1- COMPARISON BETWEEN RESUlts OF THE PROPOSED ALGORITHM (CE) AND GAMS

\begin{tabular}{|c|c|c|c|c|c|c|c|c|c|c|c|c|c|c|}
\hline \multirow{3}{*}{$\#$} & \multirow{3}{*}{$N$} & \multirow{3}{*}{$m$} & \multirow{2}{*}{\multicolumn{2}{|c|}{$\begin{array}{c}\text { GAMS } \\
\text { (Baron solver) }\end{array}$}} & \multicolumn{8}{|c|}{ Proposed Algorithm $(C E)$} & \multirow{2}{*}{\multicolumn{2}{|c|}{$\operatorname{Dev}(\%)$}} \\
\hline & & & & & \multicolumn{4}{|c|}{$F^{\text {best }}$} & \multicolumn{4}{|c|}{ Time(Sec.) } & & \\
\hline & & & Time $($ Sec $)$ & $F^{*}$ & Best & Aver & Std & $C V$ & Best & Aver & Std & $C V$ & Best & Aver \\
\hline 1 & 20 & 2 & 0.48 & 6076 & 6098 & 6106 & 4.83 & 0.079 & 6.41 & 6.64 & 0.16 & 2.42 & 0.36 & 0.50 \\
\hline 2 & 20 & 3 & 0.34 & 4652 & 4664 & 4669 & 3.20 & 0.069 & 9.33 & 9.95 & 0.84 & 8.41 & 0.27 & 0.36 \\
\hline 3 & 30 & 2 & 0.83 & 7229 & 7252 & 7270 & 7.68 & 0.106 & 7.43 & 7.71 & 0.19 & 2.49 & 0.33 & 0.57 \\
\hline 4 & 30 & 3 & 0.64 & 6146 & 6204 & 6221 & 8.33 & 0.134 & 10.59 & 11.35 & 0.49 & 4.31 & 0.94 & 1.23 \\
\hline 5 & 40 & 3 & 0.95 & 7756 & 7860 & 7879 & 13.81 & 0.175 & 11.58 & 12.39 & 0.78 & 6.28 & 1.34 & 1.58 \\
\hline 6 & 40 & 4 & 1.79 & 7259 & 7363 & 7390 & 15.97 & 0.216 & 15.41 & 16.22 & 0.58 & 3.58 & 1.43 & 1.81 \\
\hline 7 & 40 & 5 & 2.71 & 6783 & 6909 & 6945 & 15.58 & 0.224 & 19.38 & 20.82 & 0.98 & 4.73 & 1.86 & 2.39 \\
\hline 8 & 60 & 4 & 4.88 & 8183 & 8287 & 8320 & 14.30 & 0.172 & 31.92 & 32.65 & 0.43 & 1.31 & 1.26 & 1.67 \\
\hline 9 & 60 & 5 & 38.91 & 7866 & 8098 & 8115 & 10.54 & 0.130 & 38.59 & 40.64 & 1.09 & 2.68 & 2.95 & 3.16 \\
\hline 10 & 60 & 6 & 55.61 & 7702 & 7745 & 7855 & 40.36 & 0.514 & 43.00 & 49.23 & 2.97 & 6.04 & 0.56 & 1.99 \\
\hline 11 & 80 & 4 & 8.16 & 9373 & 9451 & 9486 & 19.03 & 0.201 & 37.69 & 38.91 & 1.12 & 2.88 & 0.84 & 1.21 \\
\hline 12 & 80 & 5 & 14.32 & 9345 & 9527 & 9541 & 9.08 & 0.095 & 45.16 & 48.28 & 1.35 & 2.79 & 1.94 & 2.09 \\
\hline 13 & 80 & 6 & 90.90 & 9359 & 9542 & 9600 & 21.80 & 0.227 & 56.25 & 57.33 & 0.87 & 1.52 & 1.95 & 2.57 \\
\hline 14 & 80 & 7 & 354.51 & 9431 & 9687 & 9712 & 13.99 & 0.144 & 62.85 & 65.21 & 1.27 & 1.95 & 2.71 & 2.99 \\
\hline 15 & 80 & 8 & 376.71 & 9695 & 9783 & 9839 & 31.73 & 0.322 & 75.82 & 77.64 & 1.85 & 2.38 & 0.91 & 1.49 \\
\hline 16 & 100 & 5 & 28.14 & 10489 & 10660 & 10681 & 11.61 & 0.109 & 52.01 & 53.71 & 1.27 & 2.36 & 1.63 & 1.83 \\
\hline 17 & 100 & 6 & 97.45 & 10539 & 10855 & 10892 & 17.66 & 0.162 & 63.65 & 65.02 & 1.27 & 1.95 & 2.99 & 3.34 \\
\hline 18 & 100 & 7 & 264.53 & 10830 & 11083 & 11123 & 30.93 & 0.278 & 73.13 & 75.34 & 1.51 & 2.01 & 2.34 & 2.71 \\
\hline 19 & 100 & 8 & 408.86 & 11110 & 11363 & 11417 & 26.22 & 0.230 & 79.00 & 84.34 & 2.34 & 2.77 & 2.28 & 2.76 \\
\hline 20 & 100 & 9 & 477.11 & 11461 & 11679 & 11738 & 28.64 & 0.244 & 91.15 & 93.68 & 1.85 & 1.98 & 1.90 & 2.41 \\
\hline 21 & 100 & 10 & 1046.08 & 11752 & 11993 & 12071 & 42.03 & 0.348 & 102.51 & 108.70 & 3.85 & 3.54 & 2.05 & 2.71 \\
\hline
\end{tabular}


TABLE 2- Results OF THE PROPOSED AlgORITHM (CE) FOR LARGE PROBLEMS

\begin{tabular}{|c|c|c|c|c|c|c|c|c|c|c|c|c|c|c|c|}
\hline \multirow{2}{*}{$\#$} & \multirow{2}{*}{$N$} & \multirow{2}{*}{$M$} & \multicolumn{4}{|c|}{ Time(Sec.) } & \multicolumn{4}{|c|}{$F^{\text {best }}$} & \multicolumn{3}{|c|}{ GAMS(Baron solver) } & \multicolumn{2}{|c|}{ dev. (\%) } \\
\hline & & & Best & Avr. & Std. & C.V. & Best & Avr. & Std. & C.V. & Time(Sec.) & $L B$ & $U B$ & Best & Avr. \\
\hline 1 & 200 & 10 & 134.68 & 141.23 & 4.72 & 3.34 & 17217 & 17259 & 24 & 0.14 & 995 & 15800 & 17279 & -0.36 & -0.12 \\
\hline 2 & 200 & 15 & 230.52 & 233.26 & 2.38 & 1.02 & 21110 & 21146 & 29 & 0.14 & 5007 & 18964 & 20751 & 1.73 & 1.90 \\
\hline 3 & 200 & 20 & 301.71 & 306.22 & 3.60 & 1.17 & 25657 & 25770 & 57 & 0.22 & 18000 & 22479 & 25349 & 1.22 & 1.66 \\
\hline 4 & 300 & 10 & 169.11 & 176.04 & 5.64 & 3.20 & 18550 & 18572 & 13 & 0.07 & 4829 & 17050 & 18385 & 0.90 & 1.02 \\
\hline 5 & 300 & 15 & 294.55 & 313.45 & 0.00 & 0.00 & 22884 & 22910 & 18 & 0.08 & 15085 & 20800 & 22562 & 1.43 & 1.54 \\
\hline 6 & 300 & 20 & 386.85 & 391.24 & 3.86 & 0.99 & 27388 & 27483 & 62 & 0.23 & 18000 & $N F$ & $N F$ & - & - \\
\hline 7 & 300 & 30 & 563.12 & 582.62 & 10.26 & 1.76 & 37810 & 38054 & 128 & 0.34 & 18000 & $N F$ & $N F$ & - & - \\
\hline 8 & 400 & 10 & 224.36 & 230.39 & 4.76 & 2.07 & 19218 & 19241 & 18 & 0.10 & 7148 & 17675 & 19291 & -0.37 & -0.26 \\
\hline 9 & 400 & 20 & 486.10 & 498.20 & 9.17 & 1.84 & 28571 & 28647 & 51 & 0.18 & 18000 & $N F$ & $N F$ & - & - \\
\hline 10 & 400 & 30 & 718.84 & 732.34 & 12.03 & 1.64 & 38667 & 38893 & 157 & 0.40 & 18000 & $N F$ & $N F$ & - & - \\
\hline 11 & 400 & 40 & 950.97 & 973.66 & 12.56 & 1.29 & 50516 & 50755 & 213 & 0.42 & 18000 & $N F$ & $N F$ & - & - \\
\hline 12 & 500 & 10 & 265.01 & 276.11 & 7.72 & 2.80 & 19456 & 19481 & 23 & 0.12 & 18000 & 17500 & 19549 & -0.48 & -0.35 \\
\hline 13 & 500 & 20 & 591.29 & 610.39 & 11.10 & 1.82 & 29204 & 29310 & 77 & 0.26 & 18000 & $N F$ & $N F$ & - & - \\
\hline 14 & 500 & 30 & 859.47 & 879.22 & 14.81 & 1.68 & 39596 & 39780 & 127 & 0.32 & 18000 & $N F$ & $N F$ & - & - \\
\hline 15 & 500 & 40 & 1163.28 & 1185.85 & 18.21 & 1.54 & 51290 & 51622 & 188 & 0.36 & 18000 & $N F$ & $N F$ & - & - \\
\hline 16 & 500 & 50 & 1423.45 & 1474.23 & 32.35 & 2.19 & 64494 & 64914 & 248 & 0.38 & 18000 & $N F$ & $N F$ & - & - \\
\hline 17 & 1000 & 10 & 562.31 & 586.63 & 15.47 & 2.64 & 20330 & 20352 & 18 & 0.09 & 18000 & $N F$ & $N F$ & - & - \\
\hline 18 & 1000 & 20 & 1276.87 & 1309.92 & 22.59 & 1.72 & 30889 & 30979 & 67 & 0.22 & 18000 & $N F$ & $N F$ & - & - \\
\hline 19 & 1000 & 30 & 1892.03 & 1916.06 & 21.10 & 1.10 & 42033 & 42216 & 109 & 0.26 & 18000 & $N F$ & $N F$ & - & - \\
\hline 20 & 1000 & 40 & 2485.21 & 2533.18 & 29.40 & 1.16 & 53678 & 53941 & 186 & 0.34 & 18000 & $N F$ & $N F$ & - & - \\
\hline 21 & 1000 & 50 & 3085.14 & 3131.95 & 23.37 & 0.75 & 66141 & 66384 & 191 & 0.29 & 18000 & $N F$ & $N F$ & - & - \\
\hline 22 & 1000 & 100 & 7151.88 & 7218.83 & 46.86 & 0.65 & 138602 & 139247 & 657 & 0.47 & 18000 & $N F$ & $N F$ & - & - \\
\hline
\end{tabular}

NF $=$ No feasible solution Found

According to the results from both tables it can be noted that there are very small variations in the results of the proposed $C E$ algorithm which demonstrates the robustness of our method. This claim is also shown the $C V$ values. It can also be observed that the post optimization stage was influential as it improves the solution with an average of about $1 \%$ and $2 \%$ for small/medium and large size instances, respectively while requiring a negligible extra CPU time.

For illustration purposes, we consider Stage II to show the overall reduction in the objective function values in terms of the number of iterations. Figures $1 A$ and $1 B$ present the pattern with $n=500$ and $m=40$, and $n=1000$ and $m=100$, respectively. Similar patterns are also observed for most instances. In summary, the number of iterations required to find the solution is recorded as 50 for $m \leq 10$, 60 for $10<m \leq 50$ and 70 for $m>50$. 


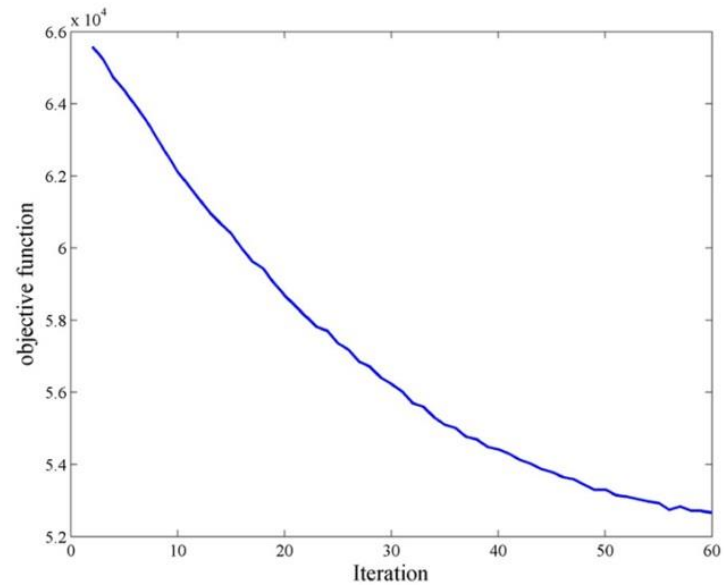

Figure 1 (A) OBJECTIVE FUNCTION VS. ITERATION NUMBER $(\mathrm{N}=500, \mathrm{M}=40)$ FOR STAGE II.

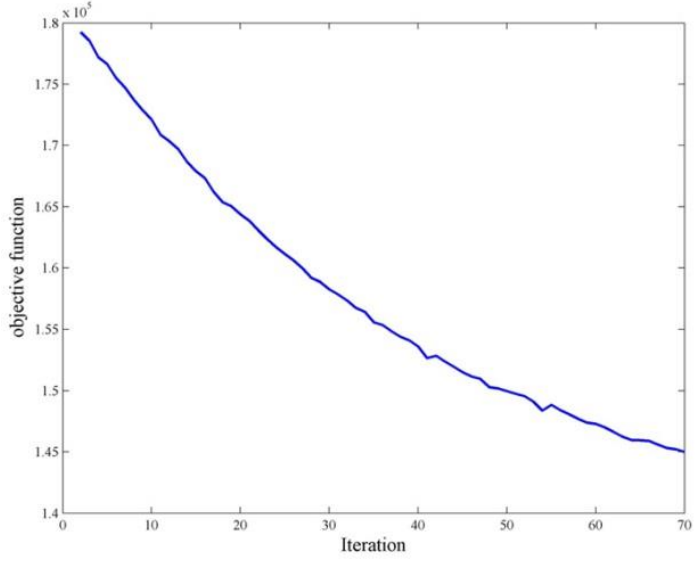

FigurE 1 (B) OBJECTIVE FUNCTION VS. ITERATION NUMBER $(\mathrm{N}=1000, \mathrm{M}=100)$ FOR STAGE II.

\section{Conclusion}

In this section, we analyze sensitivity of two parameters of the model ( $C R$ as a predetermined covering radius and $R$ which is maximum distance a facility can be located from a center of a zone to be considered as part of that zone) based on the average solution (Av.Dev.), best solution (BestDev.) and average time solution (Av.Time). Also, we recorded the results for a given instance with $n=100$ and $m=10$ and used a combination for $\mathrm{CR}$ in $[1,2]$ and $\mathrm{R}$ in $[0.2,0.6]$. The results are also reported in Table 3.

TABLE 3- SENSITIVITY ANALYSIS OF THE PROPOSED MODEL (WITH N=100 AND M=10)

\begin{tabular}{|c|c|c|c|c|c|c|c|c|c|c|c|c|c|c|}
\hline \multirow{3}{*}{ \# } & \multirow{3}{*}{$C R$} & \multirow{3}{*}{$\boldsymbol{R}$} & \multirow{2}{*}{\multicolumn{2}{|c|}{$\begin{array}{c}\text { GAMS } \\
\text { (Baron solver) }\end{array}$}} & \multicolumn{8}{|c|}{ Proposed Algorithm $(C E)$} & \multirow{2}{*}{\multicolumn{2}{|c|}{$\operatorname{Dev}(\%)$}} \\
\hline & & & & & \multicolumn{4}{|c|}{$F^{\text {best }}$} & \multicolumn{4}{|c|}{ Time(Sec.) } & & \\
\hline & & & $\operatorname{Time}(\mathrm{Sec})$ & $\boldsymbol{F}^{*}$ & Best & Aver & Std & $C V$ & Best & Aver & Std & $C V$ & Best & Aver \\
\hline 1 & 1 & 0.20 & 1721.30 & 11924 & 12076 & 12154 & 37.43 & 0.308 & 105.89 & 122.43 & 13.84 & 11.30 & 1.28 & 1.96 \\
\hline 2 & 1 & 0.40 & 1046.08 & 11752 & 12099 & 12143 & 26.27 & 0.216 & 109.36 & 122.97 & 8.49 & 6.91 & 2.95 & 3.33 \\
\hline 3 & 1 & 0.60 & 1168.92 & 11635 & 12131 & 12163 & 26.65 & 0.219 & 108.75 & 118.28 & 4.86 & 4.11 & 4.26 & 4.54 \\
\hline 4 & 1.5 & 0.20 & 1721.30 & 11924 & 12048 & 12124 & 53.38 & 0.440 & 108.41 & 111.15 & 1.86 & 1.68 & 1.04 & 1.68 \\
\hline 5 & 1.5 & 0.40 & 1046.08 & 11752 & 11992.7 & 12071 & 42.03 & 0.348 & 102.51 & 108.70 & 3.85 & 3.54 & 2.05 & 2.71 \\
\hline 6 & 1.5 & 0.60 & 1168.92 & 11635 & 11993.1 & 12117 & 65.25 & 0.539 & 105.68 & 109.98 & 2.80 & 2.54 & 3.08 & 4.14 \\
\hline 7 & 2 & 0.20 & 1721.30 & 11924 & 12065 & 12149 & 56.49 & 0.465 & 113.21 & 116.96 & 2.78 & 2.38 & 1.18 & 1.88 \\
\hline 8 & 2 & 0.40 & 1046.08 & 11752 & 12030.8 & 12117 & 49.21 & 0.406 & 111.28 & 118.66 & 5.10 & 4.29 & 2.37 & 3.10 \\
\hline 9 & 2 & 0.60 & 1168.92 & 11635 & 12096.2 & 12136 & 24.79 & 0.204 & 111.35 & 115.15 & 2.22 & 1.93 & 3.97 & 4.31 \\
\hline
\end{tabular}

Figures $2 A$ and $2 B$ present the sensitivity analysis of BestDev. and Av.Dev. values based on $\mathrm{R}$ amounts, respectively. As shown, by increasing the $R$, the BestDev. and Av.Dev. values are increased. It seems to $\mathrm{CR}=1.5$ results in better solutions which we considered for our instances. 


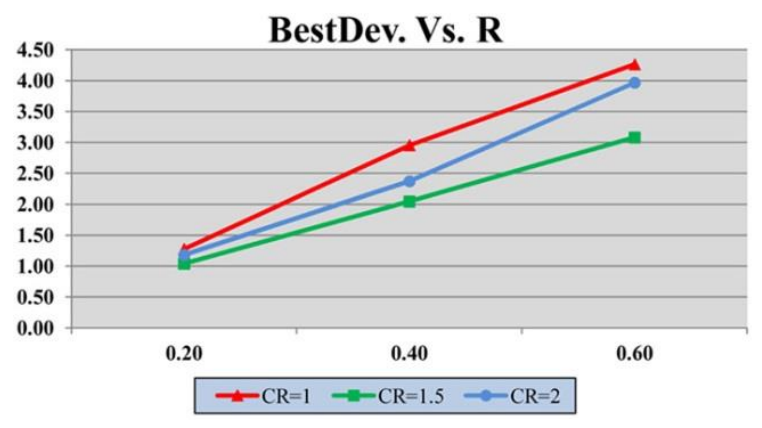

Figure 2 (A) BEST DEV. VS. R. VS. R.

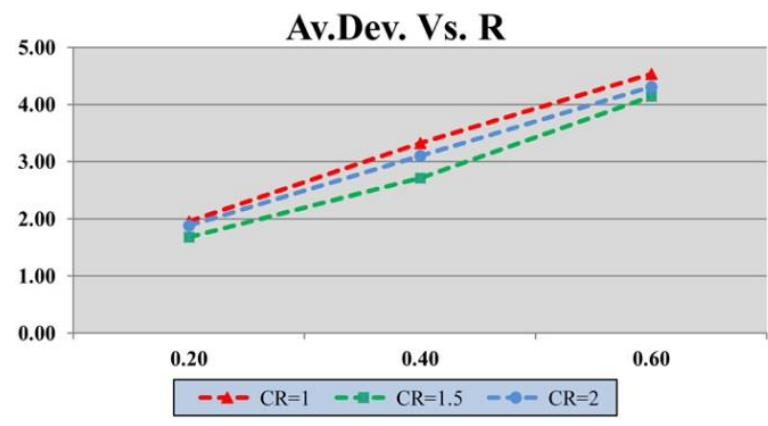

Figure 2 (B) Average DeV. vS. R

Figures $3 A$ and $3 B$ present the sensitivity analysis of BestDev. and Av.Dev. values based on CR amounts, respectively. As shown, by increasing the $C R=1.5$ and 2 results in better solution in the BestDev. and Av.Dev. values. Therefore, based on the above analysis, $R$ is more sensitive than $C R$ in providing better solution for the proposed model.

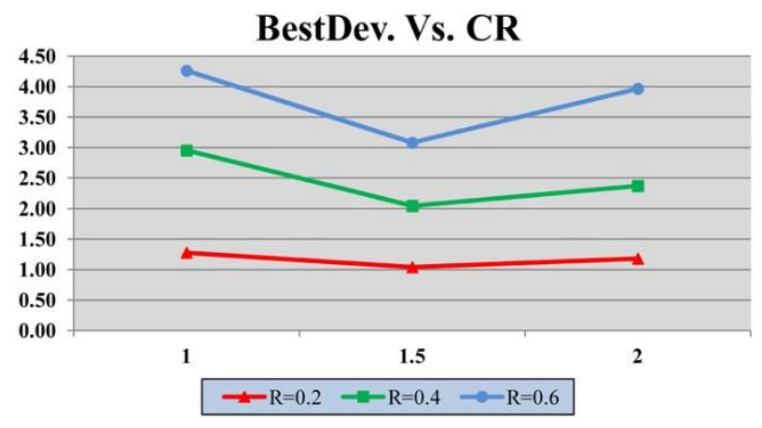

Figure 3 (A) BEST DEV. vS. CR.

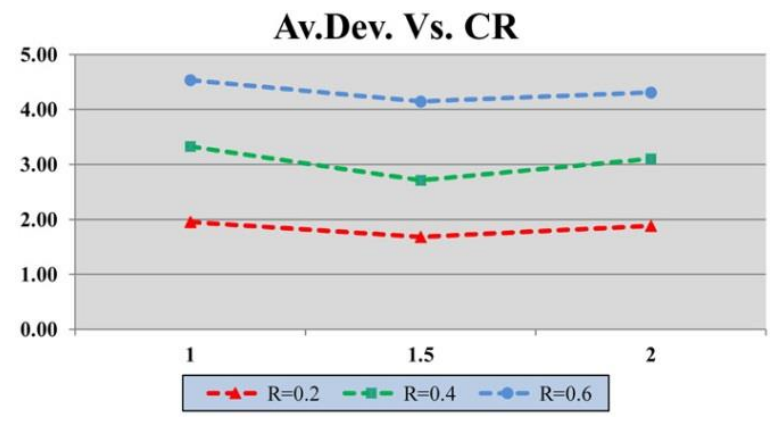

Figure 3 (B) Average Dev. vs. CR.

Figures $4 A$ and $4 B$ present the sensitivity analysis of Av.Time. values based on $R$ and $C R$ amounts, respectively. As shown, $C R$ is more sensitive than $R$ in CPU time for solving the proposed model.

Av.Time Vs. R

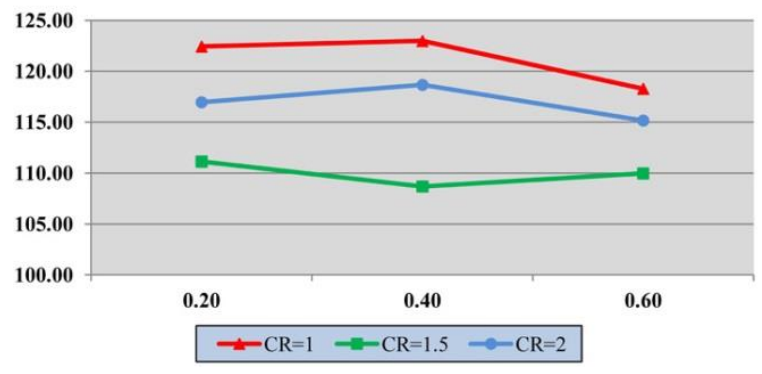

Figure 4 (A) Average Time vs. R.
Av.Time Vs. CR

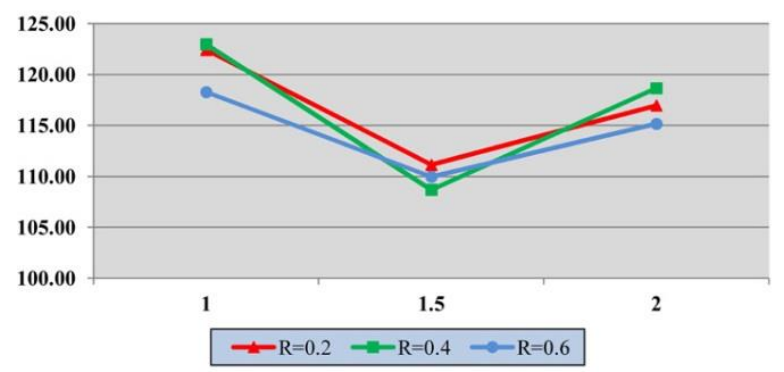

Figure 4 (B) Average Time vs. CR.

\section{Conclusion}

A capacitated multi-source Weber problem with zone-based fixed cost is investigated. A nonlinear mixed integer programming model is presented and tested on small/medium size instances using GAMS. 
An algorithm based on Cross Entropy $(C E)$ is also proposed using appropriate multivariate normal and multinomial density function for the associated stochastic problem. The proposed algorithm generated competitive results producing a deviation of less than $2 \%$ in most small/medium size instances while requiring a tiny fraction of the time needed by GAMS. In particular, for the large size instances, GAMS fails to generate lower and upper bounds in most cases even after 5 hours whereas our CE heuristic maintains its performance throughout the experiments.

The present work can be extended to include an adaptive and powerful search approach. This can be achieved by adopting a reinforced learning which continually feeds the information found in Stage III back into Stage I. The present CE meta-heuristic could also be adapted to address other related continuous location problems by appropriately defining the corresponding density functions.

\section{References}

Akyüz M. H., Altınel I. K., Öncan T. (2013), "Location and allocation based branch and bound algorithms for the capacitated multi-facility Weber problem", Annals of Operations Research, (in Press).

Bekker J., Aldrich C., (2011), "The cross-entropy method in multi-objective optimization", European Journal of Operational Research, 211, 112-121.

Brimberg J., Drezner Z., (2013), "A new heuristic for solving the p-median problem in the plane", Computers \& Operations Research, 40, 427-437.

Brimberg, J., Drezner, Z., Mladenović, N., and Salhi, S., (2014), "A New Local Search for Continuous Location Problems", European Journal of Operational Research 232, 256-265.

Brimberg J., Hansen P., Mladenovic D., Salhi S., (2008), "A Survey of Solution Methods for the Continuous Location-Allocation Problem", International journal of Operation Research, 5,1-12.

Brimberg J., Salhi S., (2005), "A Continuous Location-Allocation Problem with Zone-Dependent Fixed Cost", Annals of Operations Research, 136, 99-115.

Brimberg, J., Mladenović, N. and Salhi, S., (2004), “The Multi-Source Weber Problem with Constant Opening Cost", Journal of the Operational Research Society 55, 640-646.

Caserta M., Quinonez Rico E., (2009), "A cross entropy-Lagrangean hybrid algorithm for the multi-item capacitated lot-sizing problem with setup times", Computers \& Operations Research, 36, 530 - 548.

Caserta M., Quinonez Rico E., Marquez Uribe A.,(2008), "A cross entropy algorithm for the Knapsack problem with setups", Computers \& Operations Research, 35, 241 - 252. 
Cooper L., (1963), "Location-Allocation problems", Operations Research, 11, 3, 331-343.

Cooper L., (1972), “The transportation-location problem”, Operations Research, 20, 94-108.

de Boer P., Kroese D.P., Mannor S., Rubinstein R.Y., (2005), "A tutorial on the cross-entropy method", Annals of Operations Research, 134, 19-67.

Drezner Z., Klamroth K., Schöbel A., Wesolowsky G., (2001), The Weber problem. In: Drezner Z, Hamacher HW (eds) Facility location: applications and theory. Springer, Berlin.

DurmazE., ArasN.,Altınel I. K., (2009), "Discrete approximation heuristics for the capacitated continuous locationallocation problem with probabilistic customer locations", Computers \& Operations Research, 36, 7, 2139-2148.

Hansen P., J-F. Thisse., (1981), “ The generalized Weber-Eawls problem”. In Operational Research '81 (Hamburg, 1981), pp 569-577, North Holland, Amsterdam.

Kroese D.P., Rubinstein R.Y., (2005), "The cross-entropy method for combinatorial optimization, rare event simulation and neural computation", Annals of Operations Research, 134 (1).

Luis M., Salhi S., Nagy G., (2011), "A guided reactive GRASP for the capacitated multi-source Weber problem", Computers \& Operations Research, 38, 1014-1024.

Luis M., Salhi S., Nagy G., (2009), "Region-rejection based heuristics for the capacitated multi-source Weber problem", Computers \& Operations Research, 36, 2007 - 2017.

Mohammadi, N., Malek, M. R., and Alesheikh, A. A., (2010), "A New GA Based Solution for Capacitated Multi Source Weber Problem”, International Journal of Computational Intelligence Systems 3, 514-521.

Mousavi S. M., AkhavanNiaki S. T., (2013), "Capacitated location allocation problem with stochastic location and fuzzy demand: A hybrid algorithm", Applied Mathematical Modelling, (in Press).

Prodhon C., (2011), "A hybrid evolutionary algorithm for the periodic location-routing problem", European Journal of Operational Research, 21, 204-212.

Rubinstein R. Y., (1997), "Optimization of computer simulation models with rare events", European Journal of Operational Research, 99, 89-112.

Rubinstein R.Y., Kroese D.P., (2004), "The Cross-Entropy Method: A Unified Approach to Combinatorial Optimization", Monte-Carlo Simulation, and Machine Learning. Springer.

Shöbel A., Scholz S., (2010), "The big cube small cube solution method for multidimensional facility location problems”, Computers \& Operations Research, 37, 115-122. 